\title{
Vorkommen künstlicher Süßstoffe in deutschen Grundwässern
}

\section{Deutschlandweite Analyse und Bewertung von aktuellen Monitoringdaten zu Acesulfam, Cyclamat, Saccharin und Sucralose}

\author{
Isabel Schödl ${ }^{1}$ Falk Hilliges ${ }^{2} \mathbb{D}$ \\ Eingegangen: 21. Januar 2021 / Überarbeitet: 12. April 2021 / Angenommen: 19. Mai 2021 / Online publiziert: 17. Juni 2021 \\ ๑) Der/die Autor(en) 2021
}

\section{Zusammenfassung}

Künstliche Süßstoffe werden als Zuckeraustauschstoffe in der Lebensmittelindustrie und Landwirtschaft verwendet. Es gibt keine abschließende Bewertung über das Vorkommen in und die Auswirkungen auf Gewässer. Für künstliche Süßstoffe liegen bisher keine Grenzwerte für aquatische Umweltmedien vor. Um das Vorkommen im Grundwasser in Deutschland zu analysieren, konnten im Rahmen dieser Studie Monitoringdaten aus sieben Bundesländern und insgesamt 3311 Messstellen ausgewertet werden. Am häufigsten wurde Acesulfam, gefolgt von Cyclamat, Sucralose und Saccharin bestimmt. Acesulfam wurde mit den höchsten Konzentrationen gemessen. Für die anderen drei Süßstoffe lagen die Maximalwerte deutlich unter den Werten für Acesulfam. Für Acesulfam konnte in den letzten Jahren ein Rückgang der Konzentrationen festgestellt werden. Dies lässt sich durch den vermehrten Abbau von Acesulfam in Kläranlagen erklären. Eine Zunahme an Messwerten über der Bestimmungsgrenze, und somit ein gegenteiliger Trend, zeigte sich für Sucralose. Insgesamt ist für Deutschland die Datenlage für eine flächendeckende Zustandsbeschreibung der Belastungssituation mit Süßstoffen noch nicht ausreichend.

Schlüsselwörter Künstliche Süßstoffe · Grundwasser · Acesulfam · Cyclamat · Sucralose · Saccharin

Isabel Schödl

isabel.schoedl@uni-oldenburg.de

$\triangle$ Falk Hilliges

falk.hilliges@uba.de

1 Institut für Chemie und Biologie des

Meeres (ICBM), Universität Oldenburg,

Carl-von-Ossietzky-Straße 9-11, 26111 Oldenburg,

Deutschland

2 Abteilung Wasser und Boden, Fachgebiet „Übergreifende Angelegenheiten Wasser und Boden“, Umweltbundesamt, Wörlitzer Platz 1, 06844 Dessau-Roßlau, Deutschland 


\title{
Occurence of artificial sweeteners in German groundwater aquifers
}

Nationwide analysis and evaluation of current monitoring data on acesulfame, cyclamate, saccharine and sucralose

\begin{abstract}
Artificial sweeteners are often used as sugar substitutes but there is still no conclusive assessment of their occurrence in and their effects on water. For sweeteners there are also no threshold values for aquatic environmental media. To assess their occurrence in groundwater in Germany, monitoring data from seven federal states were evaluated as part of this study. Acesulfame was detected most frequently, followed by cyclamate, sucralose and saccharin. Acesulfame was measured at the highest concentrations. For the other sweeteners, the maximum values were below the values for acesulfame. A decrease in acesulfame concentrations could be seen over the past few years. This can be explained by the increased degradation rates in waste water treatment plants. An increase in measured values above the limit of quantification was shown for sucralose. Overall, the data situation with respect to sweeteners is not yet sufficient for a comprehensive description of the extent of pollution.
\end{abstract}

Keywords Artificial sweeteners $\cdot$ Groundwater $\cdot$ Germany $\cdot$ Acesulfame $\cdot$ Cyclamate $\cdot$ Sucralose $\cdot$ Saccharin

\section{Hintergrund}

Künstliche Süßstoffe werden weltweit in großen Mengen als Ersatz für Zucker (Saccharose) verwendet, Tendenz steigend (Research and Markets 2019). Für die Lebensmittelindustrie sind sie vor allem als Zuckeraustauschstoffe in Diätprodukten von Bedeutung (Yebra-Biurrun 2019). Sie haben eine deutlich höhere Süßkraft als Saccharose. Vier der am häufigsten eingesetzten künstlichen Süßstoffe sind Acesulfam, Cyclamat, Saccharin und Sucralose. Der Mensch scheidet den überwiegenden Teil der Süßstoffe unverdaut wieder aus und damit gelangen diese ins Abwasser (Renwick 1986; Roberts et al. 2000). Dadurch finden sich auch Anreicherungen von Süßstoffen und deren Abbauprodukten im Grundwasser. Acesulfam, Cyclamat und Saccharin sind in den 1990er-Jahren nach europäischem Lebensmittelrecht zugelassen worden. Sucralose ist in Europa seit dem Jahr 2004 zugelassen (Süßstoff Verband e. V., o.J.). Der Zeitraum zwischen der Zulassung der hier betrachteten Süßstoffe und den ersten Veröffentlichungen von Forschungsergebnissen bezüglich des Vorkommens in der Umwelt und insbesondere im Grundwasser zeigt eine große Lücke zwischen Verwendung und Erkenntnissen. Buerge et al. (2009) berichteten erstmals vom Vorkommen von Acesulfam im Grundwasser in Europa. In ihrem Untersuchungsgebiet bei Zürich konnten im Grundwasser keine weiteren der hier betrachteten Süßstoffe nachgewiesen werden. Cyclamat und Saccharin wurden erstmals von Van Stempvoort et al. (2011a, b) in einem Untersuchungsgebiet in Kanada im Grundwasser gefunden. In der Schweiz wurde Saccharin als erstes von Buerge et al. (2011a) im Grundwasser bestimmt. In Lubick (2008) wird auch die Zulassung der Süßstoffe als Nahrungszusatz ohne Kenntnisse über die Auswirkungen auf die Umwelt angesprochen. Die hier betrachteten Süßstoffe sind allerdings nicht weltweit zugelassen. Beispielsweise ist die Verwendung von Cycla- mat in den USA seit 1970 untersagt. Grund hierfür war eine an Ratten durchgeführte Studie von Oser et al. (1975), in der Cyclamat zu Blasenkrebs geführt hatte. Weitere Studien konnten allerdings keinen Zusammenhang zwischen $\mathrm{Cy}$ clamat und Krebserkrankungen feststellen (Scheurer et al. 2009). Um eine bessere Wasserlöslichkeit zu gewährleisten, werden Süßstoffe meist in der Form von Kalium- oder Natriumsalzen verwendet (LUBW 2018). Durch die hohe Wasserlöslichkeit von Süßstoffen sind Sorptionsprozesse sehr gering bis nicht vorhanden und das Medium Wasser ist für das Vorkommen der Süßstoffe in der Umwelt am relevantesten (Buerge et al. 2011a; Storck et al. 2015). Bisher wurden die Süßstoffe Acesulfam, Cyclamat, Saccharin und Sucralose im $\mathrm{Zu}$ - und Ablauf von Kläranlagen, in Klärschlamm, in Oberflächengewässern, im Grundwasser und im Trinkwasser nachgewiesen (Buerge et al. 2009; Scheurer et al. 2009; Tran et al. 2014; Tran et al. 2015). In China konnten Süßstoffe auch in der Atmosphäre an Staubpartikel haftend nachgewiesen werden (Gan et al. 2014). Nach aktuellen wissenschaftlichen Erkenntnissen sind künstliche Süßstoffe im Grundwasser, und somit auch im Trinkwasser, auch in den höchsten gemessenen Konzentrationen in Deutschland nicht gesundheitsgefährdend (LUBW 2018). Vom SCF (Scientific Committee on Food) und JECFA (Joint FAO/WHO Expert Committee on Food Additives) werden ADI-Werte (Acceptable Daily Intake) für die Süßstoffe Acesulfam, Cyclamat, Saccharin und Sucralose zwischen $5-15 \mathrm{mg} / \mathrm{kg}$ Körpergewicht pro Tag angegeben. Dies sind deutlich höhere Werte, als die Mengen, die ein Mensch pro Tag durch Trinkwasser aufnehmen kann (Kroger et al. 2006; BFR, 2014). Die gemessenen Konzentrationen an Acesulfam im Wasser sind außerdem deutlich unter der Geschmacksschwelle (Buerge et al. 2009, 2011b). Bisher gibt es in Deutschland und Europa keinen gesetzlichen Grenzwert für künstliche Süßstoffe im Grundwasser. Nach dem Europäischen Fließgewäs- 


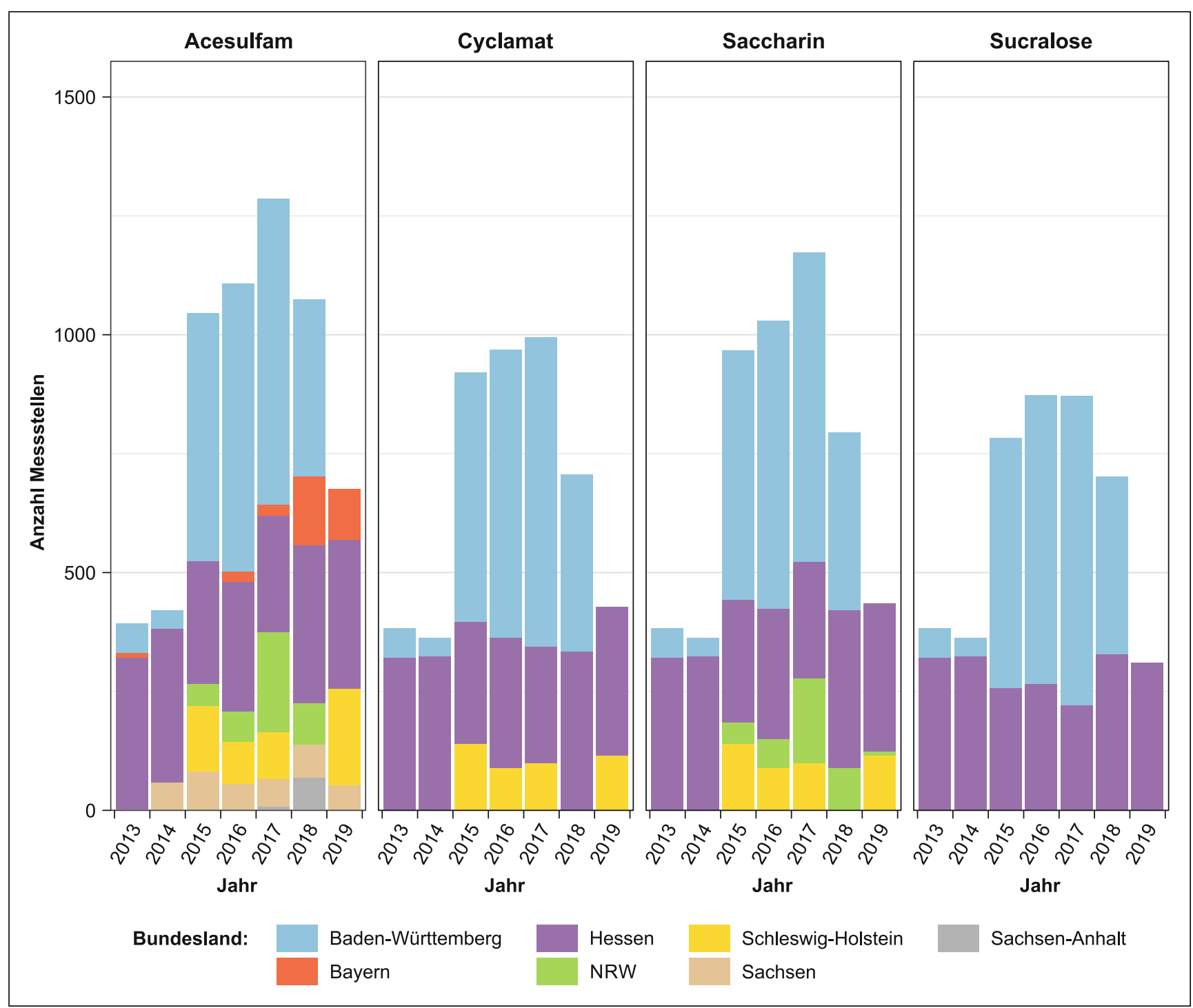

Abb. 1 Anzahl verschiedener Messstellen nach beprobtem Süßstoff, je Bundesland und Jahr

Fig. 1 Number of different monitoring sites according to the sweetener sampled, per federal state and year

sermemorandum (IAWR et al. 2013) wird allerdings für schwer abbaubare Spurenstoffe ohne bekannte Wirkungen für Fließgewässer aus denen Trinkwasser gewonnen wird ein Grenzwert von $1 \mu \mathrm{g} / \mathrm{l}$ gefordert.

\section{Eintragspfade von Süßstoffen ins Grundwasser}

Die Hauptquellen von künstlichen Süßstoffen im Grundwasser ist der Konsum von süßstoffhaltigen Lebensmitteln und die Landwirtschaft. In Deutschland wird ein Großteil des Absatzes an Süßstoffen für Softdrinks ${ }^{1}$. Mit dem Ab-

\footnotetext{
${ }^{1}$ Roth, A.: Süßstoff Verband e.V. E-Mail-Korrespondenz Mai/Juni 2020
}

wasser kommen die Stoffe über Kläranlagen in Oberflächengewässer. Ein Teil davon gelangt direkt durch Versickerung ins Grundwasser. Ebenso erfolgen Einträge durch defekte Rohrleitungen. In der Landwirtschaft werden Süßstoffe als Zusatz in der Tiernahrung verwendet. In der Europäischen Union ist Saccharin als Zusatzstoff in der Schweinezucht registriert und wird zur Entwöhnung von Ferkeln von der Muttermilch und zur Umstellung auf festes Futter verwendet (Buerge et al. 2011a; EFSA FEEDAP Panel 2018). Eine Studie von Li et al. (2020) untersuchte das Vorkommen von Süßstoffen in der Schweinemast durch eine globale Datenanalyse und einer Fallstudie in China. Wie im Zusammenhang mit der Schweinezucht erwartet, wurde Saccharin am häufigsten und mit den höchsten Konzentrationen nachgewiesen. Ein weiterer Eintragspfad in der Landwirtschaft sind Sulfonylharnstoffherbizide, bei de- 
nen Saccharin als Metabolit in den Boden und somit ins Grundwasser gelangt (Roberts et al. 1998). Beispiele für Sulfonylharnstoffherbizide sind Propoxycarbazone, Mesulfuron-methyl und Tribenuron-methyl.

\section{Material und Methoden}

Das Grundwasser wird in Deutschland aufgrund der fehlenden rechtlichen Vorgaben in der Regel nicht systematisch auf Süßstoffe beprobt. Für die Auswertung zum Vorkommen der Stoffe Acesulfam, Cyclamat, Saccharin und Sucralose im Grundwasser in Deutschland, konnten aktuelle Monitoringdaten aus sieben Bundesländern analysiert werden (Abb. 1). Die Daten umfassen Messwerte von insgesamt 3311 Messstellen und erstrecken sich über die Jahre 2013-2019. Da zu den Messstellen keine weitergehenden Stammdaten- und Lageinformationen verfügbar waren, wurde eine ganzheitliche Betrachtung der Funde der einzelnen Stoffe vorgenommen. Um einen Überblick über die zur Verfügung stehenden Daten zu erhalten, wurde die Anzahl der verschiedenen Messstellen, die je Jahr und Süßstoff in einem Bundesland beprobt wurden, berechnet.

Dabei ist zu erkennen, dass Baden-Württemberg und Hessen die über die Jahre konsistentesten Datensätze in Bezug auf die Anzahl an Messstellen haben. Für Acesulfam lagen neben diesen zwei Bundesländern auch Messwerte aus Nordrhein-Westfalen, Schleswig-Holstein, Bayern, Sachsen-Anhalt und Sachsen vor. Für Cyclamat waren nur Werte aus Baden-Württemberg, Hessen und SchleswigHolstein vorhanden. In diesen Bundesländern und in Nordrhein-Westfalen gibt es auch Messstellen, an denen Sac- charin beprobt wurde. Für Sucralose lagen nur Messwerte aus Baden-Württemberg und Hessen vor. Da es für keinen der Süßstoffe einen Grenzwert oder ein Bewertungskriterium gibt, wurden zunächst nicht die gemessenen Konzentrationen, sondern das Vorhandensein der Süßstoffe im Grundwasser analysiert und die Bestimmungshäufigkeiten je Süßstoff und Jahr, als auch über die Jahre aggregiert berechnet. Für die weiteren Analysen wurden die Daten aus den Bundesländern zu einem Datensatz zusammengefasst, um näherungsweise Aussagen für ganz Deutschland treffen zu können. Bei der Betrachtung der Fundhäufigkeiten und Konzentrationen der gemessenen Werte, wurde mit dem 90-Perzentil gearbeitet. Messwerte, die unter der Bestimmungsgrenze lagen, wurden als halbe Bestimmungsgrenze in die Berechnungen mit einbezogen.

\section{Ergebnisse}

Für Acesulfam lag die Bestimmungshäufigkeit bei 32,4\% aller Beprobungen. Die anderen Stoffe wurden deutlich weniger oft bestimmt: Cyclamat in $10,4 \%$, Saccharin in 4,4\% und Sucralose in 7,6\% der Fälle. Die gesamte Anzahl an Messwerten ist für Acesulfam am höchsten, gefolgt von Saccharin, Cyclamat und Sucralose (Abb. 2). Dies ist auch in den nach Jahren aufgeschlüsselten Daten erkennbar (Tab. 1). Die Anzahl an verschiedenen Messstellen und die Anzahl an Messwerten zeigen, dass in den Jahren 2015-2018 deutlich mehr Beprobungen für die vier Süßstoffe Acesulfam, Cyclamat, Saccharin und Sucralose durchgeführt wurden. Im Jahr 2015 steigt die Anzahl sprunghaft an, geht jedoch für das Jahr 2019 wieder zu-
Abb. 2 Bestimmungshäufigkeit und Anzahl beprobter Messstellen aggregiert über die Jahre 2013-2019

Fig. 2 Determination frequency and number of sampled measuring points aggregated (2013-2019)

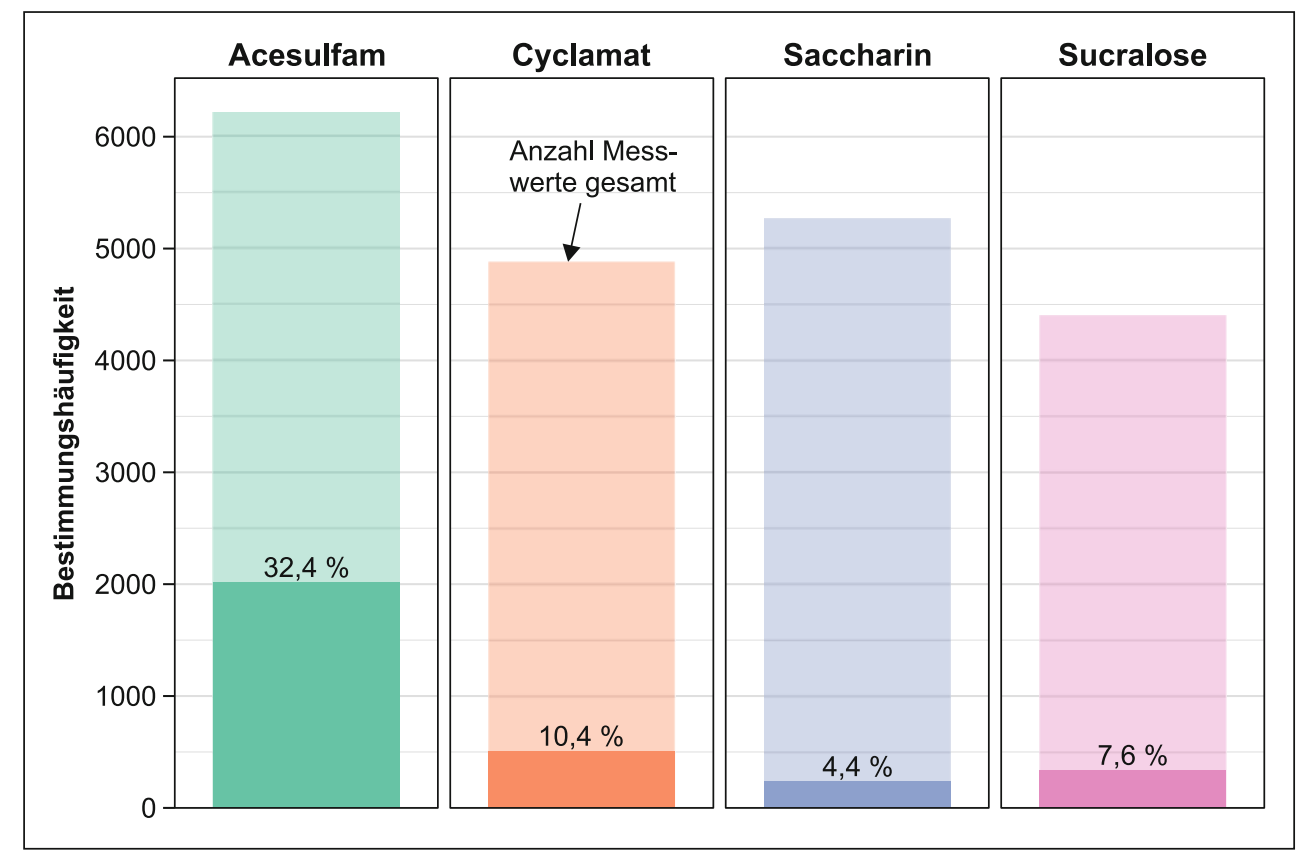


Tab. 1 Bestimmungshäufigkeit der Süßstoffe je Jahr Table 1 Detection frequency of the sweeteners per year

\begin{tabular}{|c|c|c|}
\hline Süßstoff & Jahr & Bestimmungshäufigkeit [\%] \\
\hline \multirow[t]{7}{*}{$\overline{\text { Acesulfam }}$} & 2013 & 29,6 \\
\hline & 2014 & 32,0 \\
\hline & 2015 & 27,5 \\
\hline & 2016 & 41,3 \\
\hline & 2017 & 37,9 \\
\hline & 2018 & 31,3 \\
\hline & 2019 & 19,7 \\
\hline \multirow[t]{7}{*}{ Cyclamat } & 2013 & 16,2 \\
\hline & 2014 & 11,4 \\
\hline & 2015 & 6,3 \\
\hline & 2016 & 13,4 \\
\hline & 2017 & 10,4 \\
\hline & 2018 & 8,7 \\
\hline & 2019 & 9,2 \\
\hline \multirow[t]{7}{*}{ Saccharin } & 2013 & 2,6 \\
\hline & 2014 & 3,0 \\
\hline & 2015 & 6,5 \\
\hline & 2016 & 5,3 \\
\hline & 2017 & 4,7 \\
\hline & 2018 & 2,4 \\
\hline & 2019 & 3,5 \\
\hline \multirow[t]{7}{*}{ Sucralose } & 2013 & 2,4 \\
\hline & 2014 & 2,5 \\
\hline & 2015 & 5,9 \\
\hline & 2016 & 6,9 \\
\hline & 2017 & 11,4 \\
\hline & 2018 & 6,6 \\
\hline & 2019 & 17,3 \\
\hline
\end{tabular}

rück. Unter allen vier Süßstoffen wurde Acesulfam nicht nur insgesamt, sondern auch in jedem Jahr am häufigsten bestimmt.

Bei der Betrachtung der Messwerte je Süßstoff innerhalb der Messwert-Gruppen zeigt sich, dass Acesulfam nicht nur am häufigsten bestimmt wurde, sondern auch die gemessenen Konzentrationen höher sind, als die der anderen Süßstoffe (Abb. 3 und 4). Die zweithöchsten Konzentrationen sind für Cyclamat feststellbar. Die geringsten für Saccharin. Nach Jahren aufgeteilt lassen sich Trends in der Verteilung der Messwert-Gruppen erkennen (Abb. 4). Für Acesulfam

Tab. 2 Statistische Zusammenfassung der 90-Perzentile der Messwerte je Süßstoff in $\mu \mathrm{g} / \mathrm{l}$

Table 2 Statistical summary of the 90 percentile of the measured values per sweetener in $\mu \mathrm{g} / \mathrm{l}$

\begin{tabular}{llll}
\hline Süßstoff & Maximalwert & Median & Mittelwert \\
\hline Acesulfam & 0,170 & 0,025 & 0,027 \\
Cyclamat & 0,025 & 0,005 & 0,013 \\
Saccharin & 0,025 & 0,013 & 0,015 \\
Sucralose & 0,025 & 0,025 & 0,024 \\
\hline
\end{tabular}

und Cyclamat geht der prozentuale Anteil an Messwerten in der in Klasse $\geq 1 \mu \mathrm{g} / 1$ nach 2014 deutlich zurück. Dies sind allerdings auch die Süßstoff-Jahr Kombinationen mit der geringsten Anzahl an Messwerten im Gegensatz zu den anderen Jahren. Für beide Süßstoffe lässt sich nach 2016 auch in dieser Abbildung der bereits festgestellte Trend in der Abnahme der Bestimmungshäufigkeit deutlich erkennen. Für Acesulfam gehen alle Anteile in den Gruppen mit Messwerten $>$ Bestimmungsgrenze zurück. Für Cyclamat sind nach 2016 die Anteile > Bestimmungsgrenze insgesamt rückläufig, jedoch nehmen die Anteile in den Klassen $\geq 0,1 \mu \mathrm{g} / \mathrm{l}$ bis $<1 \mu \mathrm{g} / \mathrm{l}$ und $\geq 1 \mu \mathrm{g} / \mathrm{l}$ zu. Für Cyclamat standen 2019 allerdings auch wieder weniger Messwerte zur Verfügung. Der Süßstoff mit der niedrigsten Bestimmungshäufigkeit, Saccharin, zeigt auch sehr wenige Messwerte in den größeren Messwert-Gruppen. Für Sucralose hingegen zeigt sich eine über die Jahre fast kontinuierliche Zunahme (Ausnahme in 2018) an Messwerten in den zwei oberen Messwert-Gruppen. Die Größenordnungen der gemessenen Werte unterscheiden sich zwischen den Stoffen sehr stark. Für Acesulfam beträgt der höchste gemessene Wert im gesamten Datensatz 52,6 $\mu \mathrm{g} / \mathrm{l}$. Für Cyclamat, Saccharin und Sucralose sind die Maximalwerte mit 4,0 $\mu \mathrm{g} / \mathrm{l}, 4,2 \mu \mathrm{g} / \mathrm{l}$ bzw. $3,0 \mu \mathrm{g} / \mathrm{l}$ deutlich geringer. Für Acesulfam lagen im Rohdatensatz einige sehr große Ausreißer vor. Für die anderen Süßstoffe waren derart starke Ausreißer nicht vorhanden. Deshalb wurden die Auswertungen auf Grundlage der 90Perzentile vorgenommen. Die statistischen Kenngrößen zur den wichtigsten Lageparametern sind in Tab. 2 aufgeführt.

\section{Diskussion}

Die Auswertung zeigt, dass Acesulfam in deutschen Grundwässern weit verbreitet ist und bei vermehrter Beprobung auch die Bestimmungshäufigkeit steigt. Bei Acesulfam ist ein Rückgang der prozentualen Anteile an hohen Werten erkennbar. Dies kann auf den erhöhten Abbau von Acesulfam in Kläranlagen in den letzten Jahren zurückzuführen sein und deckt sich mit den Ergebnissen von Kahl et al. (2018) und Reemtsma [2020]. In dieser Studie konnte schon für 2013-2016 gezeigt werden, dass die Konzentrationen von Acesulfam in den Flüssen Rhein und Elbe um $70-80 \%$ gesunken waren. Wie in der Einleitung erwähnt, wird Saccharin in der Schweinemast als Zusatz in Futtermitteln verwendet. Da im Rahmen dieser Arbeit keine signifikante länderübergreifende Belastung des Grundwassers mit Saccharin festgestellt werden konnte, kann darauf geschlossen werden, dass in Deutschland entweder wenig Saccharin in Futtermitteln verwendet wird oder der Eintragspfad von Saccharin über die Landwirtschaft an den betrachteten Messstellen für das Grundwasser keine Bedeutung hat. Die in den Grundwasserberichten LUBW (2018) 
Abb. 3 Messwerte der beprobten Süßstoffe nach MesswertGruppen als prozentuale Anteile aggregiert über die Jahre 2013-2019 (BG Bestimmungsgrenze)

Fig. 3 Measured values of the sampled sweeteners aggregated according to measured value groups as percentages over the years 2013-2019 (BG Limit of quantification)



und HLNUG (2018) verwendeten Daten wurden auch für diesen Bericht herangezogen. In den Grundwasserproben in Hessen wird, wie auch in Baden-Württemberg und in diesem Bericht, Acesulfam am häufigsten und mit den höchsten Werten nachgewiesen. Die anderen Stoffe werden in den Grundwasserproben aus Hessen meist unter der Bestimmungsgrenze und sonst nur knapp darüber nachgewiesen (HLNUG 2018). Die in diesem Bericht verwendeten Daten für Cyclamat, Saccharin und Sucralose über die Jahre 2013-2019 bestätigen dies nur für Saccharin. Weitere Messwerte aus Grundwasserberichten liegen aus Frankreich, dem Elsass, Rheinland-Pfalz und Basel-Stadt sowie Basel-Land vor (APRONA et al. 2018). Auch im Elsass wurde Acesulfam mit einer hohen Bestimmungshäufigkeit von $72 \%(n=100$ Messstellen) am häufigsten bestimmt. Cyclamat und Sucralose (je 18\%) und Saccharin (5\%) wurden deutlich weniger oft bestimmt, allerdings mehr als in den diesem Bericht zugrunde liegende Daten. Die höchste gemessene Acesulfamkonzentration von $1 \mu \mathrm{g} / \mathrm{l}$ im Elsass ist jedoch weit unter den in Deutschland gemessenen Werten. In Rheinland-Pfalz wurde Acesulfam an 86 Messstellen untersucht mit einer Bestimmungshäufigkeit von $24 \%$ (Maximalwert: $2 \mu \mathrm{g} / \mathrm{l})$. Die Bestimmungshäufigkeit, nicht aber der Maximalwert, liegt in der Größenordnung der Ergebnisse dieser Arbeit. In Basel und Umgebung ergab sich bei 34 Messstellen eine Bestimmungshäufigkeit von $100 \%$ mit einem Maximalwert von 1,1 $\mu \mathrm{g} / \mathrm{l}$. Saccharin wurde hier an $50 \%$ der Messstellen nachgewiesen. Die Anzahl der Messstellen war im Elsass, in Rheinland-Pfalz und in Basel im Gegensatz zu den in diesem Bericht verwendeten Daten sehr gering. Bei den Daten aus dem Elsass, Rheinland-Pfalz und Basel ergaben sich trotz geringer Messstellenanzahl sehr hohe Bestimmungshäufigkeiten. Dies könnte darauf hindeuten, dass die Messwerte sehr stark von den äußeren Bedingungen der Messstellen abhängen und geographisch nah beieinander liegende Messstellen ähnliche Ergebnisse liefern. Auch in Österreich wurden im Grundwassermonitoringprogramm 2014 an 54 ausgewählten Grundwassermessstellen Acesulfam und Sucralose als Abwasserindikatoren gemessen (BMG 2015). Die Konzentration von Acesulfam im Grundwasser überschritt an 65-68\% der Proben die Bestimmungsgrenze von $0,005 \mu \mathrm{g} / \mathrm{l}$. Die Maximalkonzentration von $2,1 \mu \mathrm{g} / \mathrm{l}$ lag auch hier deutlich unter den in diesem Bericht ausgewerteten Daten. Die Ergebnisse zu Sucralose decken sich bezüglich der Bestimmungshäufigkeit mit den Daten aus diesem Bericht. Sucralose wurde in Österreich an $8 \%$ aller Proben, aber mit einer sehr geringen Maximalkonzentration von $0,044 \mu \mathrm{g} / \mathrm{l}$, detektiert. Im landesweiten Grundwassermonitoringprogramm der Schweiz wurden für Acesulfam und Sucralose Konzentrationen von mehr als $0,1 \mu \mathrm{g} / \mathrm{l}$ festgestellt. Acesulfam wurde dabei wieder- 
Abb. 4 Relative Anteile der Messwerte der beprobten Süßstoffe nach Messwert-Gruppen über die Jahre ( $B G$ Bestimmungsgrenze)

Fig. 4 Relative amount of the measured values of the sampled sweeteners according to groups of measured values over the years, (BG Limit of quantification)

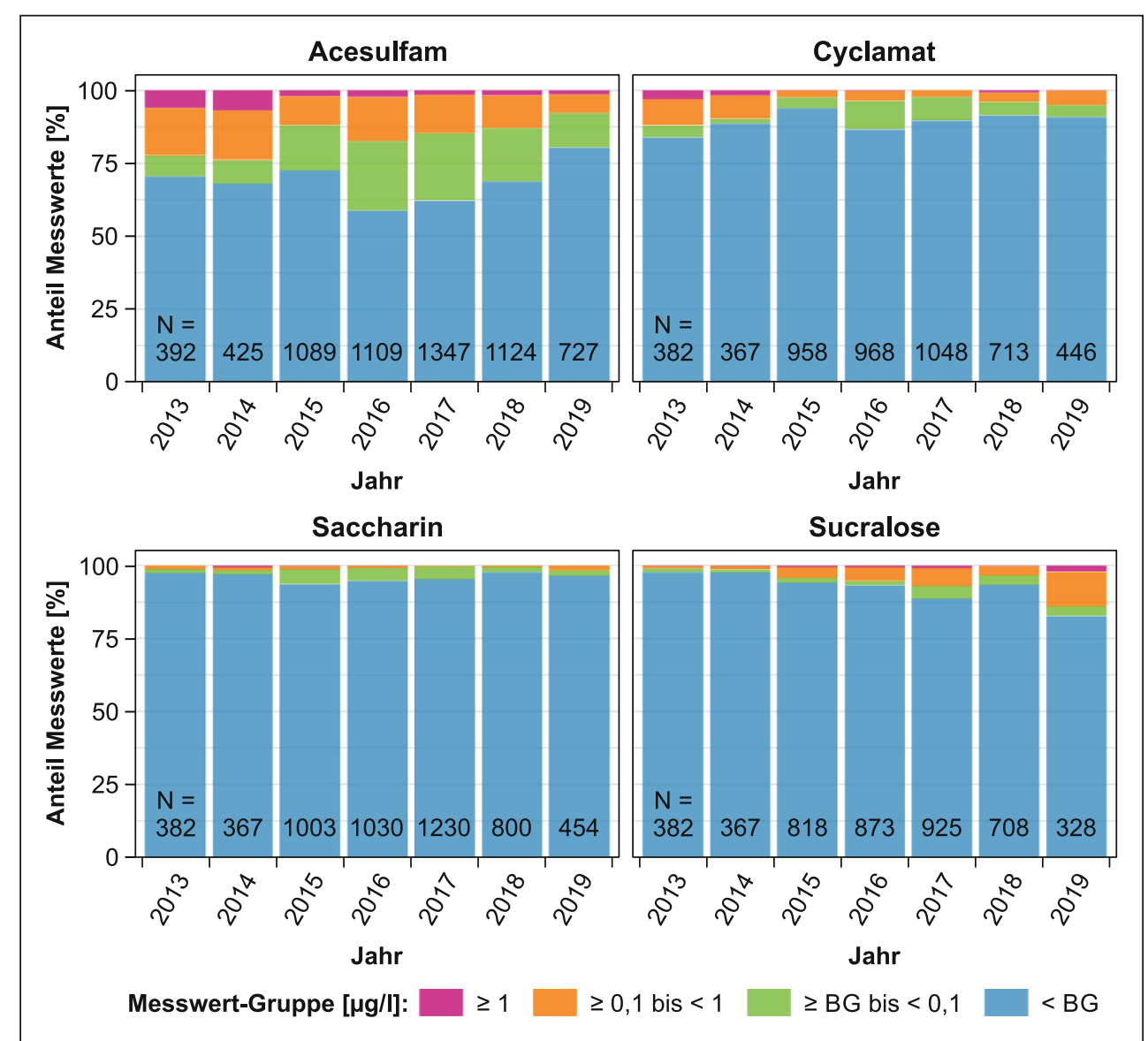

holt auch mit Konzentrationen über $1 \mu \mathrm{g} / \mathrm{l}$ gemessen (BAFU 2019).

Übergreifend wurden Acesulfam und Sucralose am häufigsten bestimmt (Buerge et al. 2009, 2011a; Scheurer et al. 2009; Ferrer und Thurman 2010; Van Stempvoort et al. 2011b; Storck et al. 2016) (siehe Luo et al. 2019 für einen Überblick). Diese Tatsache bestätigt die geringere Abbaufähigkeit von Acesulfam und Sucralose gegenüber Cyclamat und Saccharin. In der Literatur (Buerge et al. 2011a; Van Stempvoort et al. 2011b) und in diesem Bericht wurden dennoch teils hohe Messwerte für die eigentlich gut abbaubaren Süßstoffe Cyclamat und Saccharin gefunden. Dies zeigt, dass sowohl die Abbauraten als auch Sorptionsprozesse unter bestimmten Bedingungen nicht stattfinden oder gehemmt sind. Für Acesulfam wurden in Deutschland und in Kanada Werte im Grundwasser in derselben Größenordnung wie in diesem Bericht gefunden (Maximalwert: $58 \mu \mathrm{g} / \mathrm{l}$ bzw. 33,6 $\mu$ g/l) (Scheurer et al. 2009; Van Stempvoort et al. 2011b).

Eine Auswertung nach den Standorten der Messstellen und Umwelt-/Bodenbedingungen war mit den für diese Arbeit zur Verfügung stehenden Daten nicht möglich. Dennoch hat dies großen Einfluss auf die Interpretation der Da- ten und sollte in weitergehenden Analysen mit betrachtet werden.

$11 \%$ der Messstellen der in diesem Bericht verwendeten Daten wurden nur einmal in den Jahren 2013-2019 beprobt. $67 \%$ der Messstellen wurden zwar mehrmals beprobt, allerdings nur innerhalb eines Jahres. Für eine aussagekräftige Trendanalyse werden konsistentere Datensätze benötigt, in der alle Messstellen über alle Jahre beprobt werden und eine höhere zeitliche Auflösung vorweisen. Die vielen Einzelmessungen in den vorliegenden Daten zeigen, dass es sinnvoll ist, die Daten aus den Bundesländern zusammengefasst für Deutschland zu analysieren, wie es im Rahmen dieser Arbeit durchgeführt wurde. Anzumerken ist auch, dass für Sucralose nur Daten aus zwei Bundesländern vorlagen. In diesen beiden Bundesländern wurde allerdings Sucralose an vielen Messstellen beprobt. Die Inhomogenität in den Messstellen erklärt in Teilen auch die unterschiedliche Streuung der Messwerte über die Jahre. Da die Verweilzeiten von Grundwasser in der Regel mehrere Jahre, teils Jahrzehnte betragen, bedingen sich die Messungen über die Jahre. Das Fehlen eines landesweiten Datensatzes findet sich auch in anderen Ländern, wie beispielsweise der Schweiz (Stand 2016). Daraufhin wurde in der Schweiz die 
Maßnahme ergriffen, Acesulfam ab 2015 in das Langzeitmonitoring des Grundwassers aufzunehmen (BAFU 2019).

\section{Fazit}

In den bisherigen Untersuchungen zu Süßstoffen im Grundwasser in Deutschland, wurden immer ausschließlich regionale bzw. lokale Aspekte betrachtet. Mithilfe der für diese Arbeit verwendeten Daten konnte erstmals eine Beschreibung der aktuellen Belastungssituation über mehrere Bundesländer durchgeführt werden. Dennoch ist aufgrund einer fehlenden übergreifenden Monitoringstrategie eine valide Gesamtaussage zur Belastungssituation in Deutschland nicht möglich. Eine Ursache für die heterogene Überwachung in den Bundesländern ist das Fehlen regulatorischer Schwellenwerte. Auch in anderen Ländern ist das zu beobachten. Auch wenn von Süßstoffen keine nachgewiesene toxikologische Gefährdung ausgeht, so sind es doch für das Trinkwasser unerwünschte Stoffe, deren Regulierung zumindest geprüft werden sollte.

Funding Open Access funding enabled and organized by Projekt DEAL.

Open Access Dieser Artikel wird unter der Creative Commons Namensnennung 4.0 International Lizenz veröffentlicht, welche die Nutzung, Vervielfältigung, Bearbeitung, Verbreitung und Wiedergabe in jeglichem Medium und Format erlaubt, sofern Sie den/die ursprünglichen Autor(en) und die Quelle ordnungsgemäß nennen, einen Link zur Creative Commons Lizenz beifügen und angeben, ob Änderungen vorgenommen wurden.

Die in diesem Artikel enthaltenen Bilder und sonstiges Drittmaterial unterliegen ebenfalls der genannten Creative Commons Lizenz, sofern sich aus der Abbildungslegende nichts anderes ergibt. Sofern das betreffende Material nicht unter der genannten Creative Commons Lizenz steht und die betreffende Handlung nicht nach gesetzlichen Vorschriften erlaubt ist, ist für die oben aufgeführten Weiterverwendungen des Materials die Einwilligung des jeweiligen Rechteinhabers einzuholen.

Weitere Details zur Lizenz entnehmen Sie bitte der Lizenzinformation auf http://creativecommons.org/licenses/by/4.0/deed.de.

\section{Literatur}

APRONA, HLNUG, LfU-RP, SGD-Süd, LUBW, AUE-BS, AUE-BL: ERMES-Rhein 2016 - Entwicklung der Grundwasserqualität im Oberrheingraben von Basel bis Mainz-Wiesbaden und Empfehlungen zur Reduktion historischer und neuartiger anthropogener Schadstoffe. Techn. Ber. APRONA, Colmar (2018). https://www. aprona.net/uploads/pdf/ERMES-Rhin/ERMES_Rhin_rapport_ technique.pdf, Zugegriffen: 4. Juni 2020

BAFU: Zustand und Entwicklung Grundwasser Schweiz. Ergebnisse der Nationalen Grundwassserbeobachtung NAQUA, Stand 2016. Bundesamt für Umwelt (BAFU). Bern, Schweiz (2019). https://www.bafu.admin.ch/dam/bafu/de/dokumente/wasser/uzumwelt-zustand/zustandundentwicklunggrundwasserschweiz. pdf.download.pdf/UZ-1901-D_NAQUA.pdf, Zugegriffen: 18. März 2021
BFR: Bundesinstitut Für Risikobewertung: Bewertung von Süßstoffen und Zuckeraustauschstoffen: Hintergrundinformation $\mathrm{Nr}$. 025/2014 des BfR vom 1. Juli 2014 (2014). https://www.bfr. bund.de/cm/343/bewertung_von_suessstoffen.pdf, Zugegriffen: 29. Mai 2020

BMG: Monitoringprogramm von Pharmazeutika und Abwasserindikatoren in Grund- und Trinkwasser. Forschungsprojekt - Endbericht, Juni 2015. Bundesministerium für Gesundheit (BMG). Wien, Österreich (2015). https://www.verbrauchergesundheit.gv. at/lebensmittel/trinkwasser/Monitoringprogramm_von_Pharma zeutika_und_Abwasserindikatoren.pdf?7vj8g3. Zugegriffen: 18. März 2021

Buerge, I.J., Buser, H.R., Kahle, M., Müller, M.D., Poiger, T.: Ubiquitous occurrence of the artificial sweetener acesulfame in the aquatic environment: An ideal chemical marker of domestic wastewater in groundwater. Environ. Sci. Technol. 43, 4381-4385 (2009)

Buerge, I.J., Keller, M., Buser, H.R., Müller, M.D., Poiger, T.: Saccharin and other artificial sweeteners in soils: estimated inputs from agriculture and households, degradation, and leaching to groundwater. Environ. Sci. Technol. 45, 615-621 (2011a)

Buerge, I.J., Poiger, T.: Acesulfam: ein künstlicher Süßstoff als Abwasserindikator. Nachr. Chem. 59, 1084-1086 (2011b)

EFSA FEEDAP Panel (EFSA Panel on Additives and Products or Substances used in Animal Feed), Rychen, G., Aquilina, G., Azimonti, G., Bampidis, V., Bastos, M.L., Bories, G., Cocconcelli, P.S., Flachowsky, G., Gropp, J., Kolar, B., Kouba, M., Lopez-Alonso, M., López Puente, S., Mantovani, A., Mayo, B., Ramos, F., Saarela, M., Villa, RE., Wallace, RJ., Wester, P., Brantom, P., Dusemund, B., Van Beelen, P., Westendorf, J., Gregoretti, L., Manini, P., Chesson, A.: Scientific Opinion on the safety and efficacy of sodium saccharin when used as a feed flavour for piglets, pigs for fattening, calves for rearing and calves for fattening. EFSA Journal 16 (2018)

Ferrer, I., Thurman, E.M.: Analysis of sucralose and other sweeteners in water and beverage samples by liquid chromatography/timeof-flight mass spectrometry. J. Chromatogr. A 1217, 4127-4134 (2010)

Gan, Z., Sun, H., Yao, Y., Zhao, Y., Li, Y., Zhang, Y., Hu, H., Wang, R.: Distribution of artificial sweeteners in dust and soil in China and their seasonal variations in the environment of Tianjin. Science of The Total Environment 488-489, 168-175 (2014)

HLNUG: Grundwasserbeschaffenheitsbericht 2017. Hessisches Landesamt für Naturschutz Umwelt und Geologie (HLNUG) (2018). https://www.hlnug.de/fileadmin/dokumente/wasser/grundwasser/ artikel/Grundwasserbeschaffenheitsbericht_2017.pdf, Zugegriffen: 5. Mai 2020

IAWR, RIWA, AWE, IAWD, AWWR: Europäisches Fließgewässermemorandum zur qualitativen Sicherung der Trinkwassergewinnung Memorandum regarding the protection of European rivers and watercourses in order to protect the provision of drinking water (2013). https://www.awwr.de/fileadmin/awwr_de/content/ download/efg_memorandum_2013.pdf, Zugegriffen: 5. Mai 2020

Kahl, S., Kleinsteuber, S., Nivala, J., Van Afferden, M., Reemtsma, T.: Emerging biodegradation of the previously persistent artificial sweetener acesulfame in biological wastewater treatment. Environ. Sci. Technol. 52, 2717-2725 (2018)

Kroger, M., Meister, K., Kava, R.: Low-calorie sweeteners and other sugar substitutes: a review of the safety issues. Compr. Rev. Food Sci. Food Saf. 5, 35-47 (2006)

Li, D., Yao, Y., Sun, H., Wang, Y., Pu, J., Calderón, R., Alder, A.C., Kannan, K.: Artificial sweeteners in pig feed: a worldwide survey and case study in pig farms in Tianjin, China. Environ. Sci. Technol. 54, 4059-4067 (2020)

Lubick, N.: Artificial sweetener persists in the environment. Environ. Sci. Technol. 42, 3125 (2008)

LUBW: Grundwasserüberwachungsprogramm. Ergebnisse der Beprobung 2017. Landesanstalt für Umwelt Baden-Württemberg 
(LUBW), Karlsruhe (2018). https://pd.lubw.de/89772, Zugegriffen: 29. Mai 2020

Luo, J., Wu, L., Zhang, Q., Wu, Y., Fang, F., Feng, Q., Li, C., Xue, Z., Cao, J.: Review on the determination and distribution patterns of a widespread contaminant artificial sweetener in the environment. Environ. Sci. Pollut. Res. 26, 19078-19096 (2019)

Oser, B.L., Carson, S., Cox, G.E., Vogin, E.E., Sternberg, S.S.: Chronic toxicity study of cyclamate: Saccharin $(10: 1)$ in rats. Toxicology 4, 385-386 (1975)

Reemtsma, T.: Untersuchung zur Elimination von Acesulfam und anderen Spurenstoffen. Toshovski et al, 2020: Prioritäre Stoffe in kommunalen Kläranlagen - Ein deutschlandweit harmonisiertes Vorgehen. UBA-Texte. Unpubliziert (2020)

Renwick, A.G.: The metabolism of intense sweeteners. Xenobiotica 16, 1057-1071 (1986)

Research and markets: artificial sweetener market - forecasts from 2019 to 2024 (2019). https://www.researchandmarkets.com/ reports/4801618/artificial-sweetener-market-forecastsfrom-2019, Zugegriffen: 17. Juni 2020

Roberts, A., Renwick, A.G., Sims, J., Snodin, D.J.: Sucralose metabolism and pharmacokinetics in man. Food Chem. Toxicol. 38, $31-41(2000)$

Roberts, T.R., Hutson, D.H., Lee, P.W., Nicholls, P.H., Plimmer, J.R.: Metabolic pathways of agrochemicals. Part 1: herbicides and plant growth regulators. The Royal Society of Chemistry, Cambridge, S. 872 (1998)

Scheurer, M., Heinz, J.B., Lange, F.T.: Analysis and occurrence of seven artificial sweeteners in German waste water and surface water and in soil aquifer treatment (SAT). Anal Bioanal Chem 394, 1585-1594 (2009)
Storck, F.R., Brauch, H-J., Skark, C., Remmler, F., Zullei-Seibert, N.: Acesulfam - ein universeller Tracer?, ewp - DVGW energie I wasser-praxis 66, 26-31 (2015)

Storck, F.R., Skark, C., Remmler, F., Brauch, H.-J.: Environmental fate and behavior of acesulfame in laboratory experiments. Water Sci. Technol. 74, 2832-2842 (2016)

Süßstoff Verband e.V.: Zugelassene Süßstoffe im Überblick. SüßstoffVerband e.V. https://www.suessstoff-verband.info/suessstoffwissen/suessstoffe-ueberblick/, Zugegriffen: 29. Mai 2020

Tran, N.H., Hu, J., Li, J., Ong, S.L.: Suitability of artificial sweeteners as indicators of raw wastewater contamination in surface water and groundwater. Water Research 48, 443-456 (2014)

Tran, N.H., Gan, J., Nguyen, V.T., Chen, H., You, L., Duarah, A., Zhang, L., Gin, K.Y-H.:Sorption and biodegradation of artificial sweeteners in activated sludge processes. Bioresource Technology 197, 329-338 (2015)

Van Stempvoort, D.R., Robertson, W.D., Brown, S.J.: Artificial sweeteners in a large septic plume. Ground Water Monit. Remediat. 31, 95-102 (2011a)

Van Stempvoort, D.R., Roy, J.W., Brown, S.J., Bickerton, G.: Artificial sweeteners as potential tracers in groundwater in urban environments. J. Hydrol. Reg. Stud. 401, 126-133 (2011b)

Yebra-Biurrun, M.C.: Food and nutritional analysis I Sweeteners, 3. Aufl. Encyclopedia of Analytical Science., S. 471-481 (2019)

Hinweis des Verlags Der Verlag bleibt in Hinblick auf geografische Zuordnungen und Gebietsbezeichnungen in veröffentlichten Karten und Institutsadressen neutral. 